\title{
Some New Rational Gushel Fourfolds
}

\author{
Giovanni Staglianò®o
}

\begin{abstract}
We provide explicit equations and parameterizations of some new rational Gushel-Mukai fourfolds of special type.
\end{abstract}

Mathematics Subject Classification. 14J35, 14J45, 68W30, 14Q10.

\section{Introduction}

The problem of rationality of Fano fourfolds, with particular regard to the cases of cubic fourfolds and Gushel-Mukai fourfolds, dates back to classical works; see e.g. [10,23,26] (see also Table 2 below). Despite the great attention received and the numerous results obtained, this is still an open problem for the general fourfold. For instance, no examples of cubic fourfolds as well as of Gushel-Mukai fourfolds are known to be irrational, and there are not so many constructions of rational examples. The contribution of this paper is in the construction of some new special examples of rational Gushel-Mukai fourfolds. This is achieved by determining their equations through calculations with Macaulay2 [13], using mainly the packages SpecialFanoFourfolds [34] and Cremona [31]. In particular, these packages provide the tools needed to verify the claims in the paper.

Recall that by a result of Mukai [24] (extending to all dimensions a result of Gushel proved in [14] only in dimension three), a complex smooth prime Fano fourfold $X$ of degree 10 and genus 6, also known as Gushel-Mukai fourfold, can be embedded in $\mathbb{P}^{8}$ as a quadratic section of a 5-dimensional linear section $Y \subset \mathbb{P}^{8}$ of the cone $C(\mathbb{G}(1,4)) \subset \mathbb{P}^{10}$ over the Grassmannian $\mathbb{G}(1,4) \subset \mathbb{P}^{9}$ of lines in $\mathbb{P}^{4}$. There are two cases:

- either $Y$ does not contain the vertex of the cone $C(\mathbb{G}(1,4))$, in which case $Y$ is isomorphic to a hyperplane section of $\mathbb{G}(1,4)$, and we have an embedding $\gamma_{X}: X \hookrightarrow \mathbb{G}(1,4)$;

- or otherwise $Y$ is isomorphic to a cone over a 4-dimensional linear section $Y_{0}$ of $\mathbb{G}(1,4)$, and we have a double cover $\gamma_{X}: X \rightarrow Y_{0} \subset \mathbb{G}(1,4)$.

The fourfolds in the second case are called Gushel fourfolds, and they are specializations of fourfolds in the first case, called Mukai (or ordinary) fourfolds. In both cases, the map $\gamma_{X}$ from $X$ to $\mathbb{G}(1,4)$ is called the Gushel map. 
By results proved in [3] (see also [4-6]), Fano fourfolds as above are parameterized (up to isomorphism) by the points of a coarse moduli space $\mathcal{M}_{4}$ of dimension 24, where the Gushel fourfolds correspond to the points of a closed irreducible subvariety $\mathcal{M}_{4}^{G} \subset \mathcal{M}_{4}$ of codimension 2. A fourfold $[X] \in \mathcal{M}_{4}$ is said to be special (or Hodge-special) if it contains a surface whose cohomology class does not lie in $\gamma_{X}^{*}\left(H^{4}(\mathbb{G}(1,4), \mathbb{Z})\right)$; equivalently, $[X]$ is special if and only if $\operatorname{rk}\left(H^{2,2}(X) \cap H^{4}(X, \mathbb{Z})\right) \geq 3$. A fourfold which corresponds to a very general point in $\mathcal{M}_{4}$ or in $\mathcal{M}_{4}^{G}$ is not special. More precisely, special fourfolds are parametrized by an infinite countable union of hypersurfaces $\bigcup_{d}\left(\mathcal{M}_{4}\right)_{d} \subset \mathcal{M}_{4}$, labelled by the integers $d \geq 10$ with $d \equiv 0$, 2 , or $4(\bmod 8)$. If $d \equiv 0(\bmod 4)$ then the hypersurface $\left(\mathcal{M}_{4}\right)_{d}$ is irreducible, while if $d \equiv 2(\bmod 8)$ then it is the union of two irreducible components $\left(\mathcal{M}_{4}\right)_{d}^{\prime} \cup\left(\mathcal{M}_{4}\right)_{d}^{\prime \prime}$. When a fourfold $[X]$ corresponds to a very general point of a component of $\left(\mathcal{M}_{4}\right)_{d}$, then the lattice $H^{2,2}(X) \cap H^{4}(X, \mathbb{Z})$ has rank 3 and discriminant $d$. One says that $X$ has discriminant $d$ if $[X] \in\left(\mathcal{M}_{4}\right)_{d}$.

Suppose we have a fourfold $[X] \in \mathcal{M}_{4}$ containing an irreducible surface $S$ of degree $\operatorname{deg}(S)$ and (sectional) genus $g(S)$, which has smooth normalization and only a finite number $\delta$ of nodes as singularities. Let $a \sigma_{3,1}+b \sigma_{2,2}$ be the class of $\gamma_{X *}(S)$ in the Chow ring of $\mathbb{G}(1,4)$. The double point formula (see [12, Theorem 9.3] and also [3, Section 7]) gives the value of the self-intersection of $S$ in $X$ :

$$
(S)_{X}^{2}=3 a+4 b-2 \operatorname{deg}(S)+4 g(S)-12 \chi\left(\mathcal{O}_{S}\right)+2 K_{S}^{2}-4+2 \delta .
$$

Thus we have that $[X] \in\left(\mathcal{M}_{4}\right)_{d}$, where $d$ is the discriminant of the lattice spanned by $\left(\gamma_{X}^{*}\left(\sigma_{1,1}\right), \gamma_{X}^{*}\left(\sigma_{2}\right),[S]\right)$, that is

$$
d=\operatorname{disc}\left(\begin{array}{c|c|c|c} 
& \gamma_{X}^{*}\left(\sigma_{1,1}\right) & \gamma_{X}^{*}\left(\sigma_{2}\right) & {[S]} \\
\hline \gamma_{X}^{*}\left(\sigma_{1,1}\right) & 2 & 2 & b \\
\hline \gamma_{X}^{*}\left(\sigma_{2}\right) & 2 & 4 & a \\
\hline[S] & b & a & (S)_{X}^{2}
\end{array}\right)=4(S)_{X}^{2}-2 a^{2}+4 a b-4 b^{2} .
$$

Moreover, when $d \equiv 2(\bmod 8)$, we have that $[X] \in\left(\mathcal{M}_{4}\right)_{d}^{\prime}$ if $a+b$ is even, and $[X] \in\left(\mathcal{M}_{4}\right)_{d}^{\prime \prime}$ if $b$ is even.

For some values of the discriminant $d$, a fourfold $[X] \in\left(\mathcal{M}_{4}\right)_{d}$ has an associated K3 surface of degree $d$; and for others, it has an associated cubic fourfold of discriminant $d$; see [3, Section 6] for precise definitions. The first values for which there is an associated K3 surface are:

$$
10,12,16,18,20,24,26,28,32,34,36,40,42,44,48,50,52,56,58,60, \ldots
$$

while, the first values for which there is an associated cubic fourfold are:

$$
10,12,16,18,20,24,26,28,32,34,36,40,42,44,48,50,52,56,58,60, \ldots
$$

The notion of associated K3 surface leads to the following conjecture, which is analogous to the so-called Kuznetsov conjecture for the rationality of cubic fourfolds (see $[1,2,17,21,22,27,28]$ ): 
Conjecture. A fourfold $[X] \in \mathcal{M}_{4}$ is rational if and only if it has an associated $K 3$ surface, that is $[X]$ belongs to the infinite union:

$$
\left(\mathcal{M}_{4}\right)_{10}^{\prime} \cup\left(\mathcal{M}_{4}\right)_{10}^{\prime \prime} \cup\left(\mathcal{M}_{4}\right)_{20} \cup\left(\mathcal{M}_{4}\right)_{26}^{\prime} \cup\left(\mathcal{M}_{4}\right)_{26}^{\prime \prime} \cup\left(\mathcal{M}_{4}\right)_{34}^{\prime} \cup\left(\mathcal{M}_{4}\right)_{34}^{\prime \prime} \cup \cdots
$$

The rationality for fourfolds in $\left(\mathcal{M}_{4}\right)_{10}^{\prime}$ is easy to show (see [3, Proposition 7.3], [29, Section 4.4], and also [19, Example 1.1]), and moreover the associated K3 surface of degree 10 appears naturally in the construction of the birational map $\mathbb{P}^{4} \rightarrow X$. The rationality for the fourfolds in $\left(\mathcal{M}_{4}\right)_{10}^{\prime \prime}$ is classical: it is achieved by Roth in [26] as a consequence of a result of Enriques $[9,30]$; see also [3, Proposition 7.5]. In this last case, however, the role of the associated K3 surface is not so clear. In the recent paper [19], it is showed that a general fourfold in $\left(\mathcal{M}_{4}\right)_{20}$ (and hence every by the main result in [20]) is rational. Again, the associated K3 surface of degree 20 appears in the explicit construction leading to rationality. In conclusion, we have that every fourfold in the first three components of $(0.3)$ is rational.

Restricting attention to the case of Gushel fourfolds, we point out that using the same method presented in [32], just by replacing the role of the smooth cubic scroll surface in [32, Table 2] with that of a cone over a twisted cubic curve, one can find explicit Gushel fourfolds in $\left(\mathcal{M}_{4}\right)_{10}^{\prime},\left(\mathcal{M}_{4}\right)_{10}^{\prime \prime}$, and $\left(\mathcal{M}_{4}\right)_{20}$. In particular, the following three intersections

$$
\left(\mathcal{M}_{4}\right)_{10}^{\prime} \cap \mathcal{M}_{4}^{G}, \quad\left(\mathcal{M}_{4}\right)_{10}^{\prime \prime} \cap \mathcal{M}_{4}^{G}, \quad\left(\mathcal{M}_{4}\right)_{20} \cap \mathcal{M}_{4}^{G}
$$

are not empty, and hence they parametrize rational fourfolds.

As far as the author knows, no other fourfolds in $\mathcal{M}_{4}$ as well as in $\mathcal{M}_{4}^{G}$ are known to be rational. In the following of this paper, we explain how to find explicit equations and parameterizations of rational fourfolds in $\left(\mathcal{M}_{4}\right)_{26}^{\prime \prime} \cap \mathcal{M}_{4}^{G}$ and $\left(\mathcal{M}_{4}\right)_{26}^{\prime \prime} \backslash \mathcal{M}_{4}^{G}$; see also Table 1 for a summary.

\section{Construction of New Rational Gushel Fourfolds}

In this section, we construct rational fourfolds in $\left(\mathcal{M}_{4}\right)_{26}^{\prime \prime} \cap \mathcal{M}_{4}^{G}$. Here we briefly summarize the construction. In Sect. 1.1, starting with a general cubic fourfold $C$ in $\mathcal{C}_{26}$, we explain how to determine the equations of a smooth surface $S \subset \mathbb{P}^{8}$ of degree 17 and sectional genus 11, which is isomorphic to a triple projection of a minimal K3 surface of degree 26 in $\mathbb{P}^{14}$. In Sect. 1.2, we embed the surface $S$ into a smooth quadratic section $X$ of a cone $Y$ over a smooth 4 -dimensional linear section of $\mathbb{G}(1,4)$. Then we deduce that $X$ is a fourfold in $\left(\mathcal{M}_{4}\right)_{26}^{\prime \prime} \cap \mathcal{M}_{4}^{G}$ since $\gamma_{X *}([S])=11 \sigma_{3,1}+6 \sigma_{2,2}$ in the Chow ring of $\mathbb{G}(1,4)$. In Sect. 1.3, we remark that the fourfold $X$ may not be a general point in $\left(\mathcal{M}_{4}\right)_{26}^{\prime \prime} \cap \mathcal{M}_{4}^{G}$. In Sect. 1.4, we illustrate two different methods to deduce that the surface $S$ admits inside the cone $Y$ a congruence of 9 -secant rational normal quintic curves: through a general point of $Y$ there passes a unique rational normal quintic curve which is 9 -secant to $S$ and contained in $Y$. In Sect. 1.5, we show how from this congruence of quintic curves, using in an essential way that $Y$ is not smooth, we can deduce that $X$ is rational. 


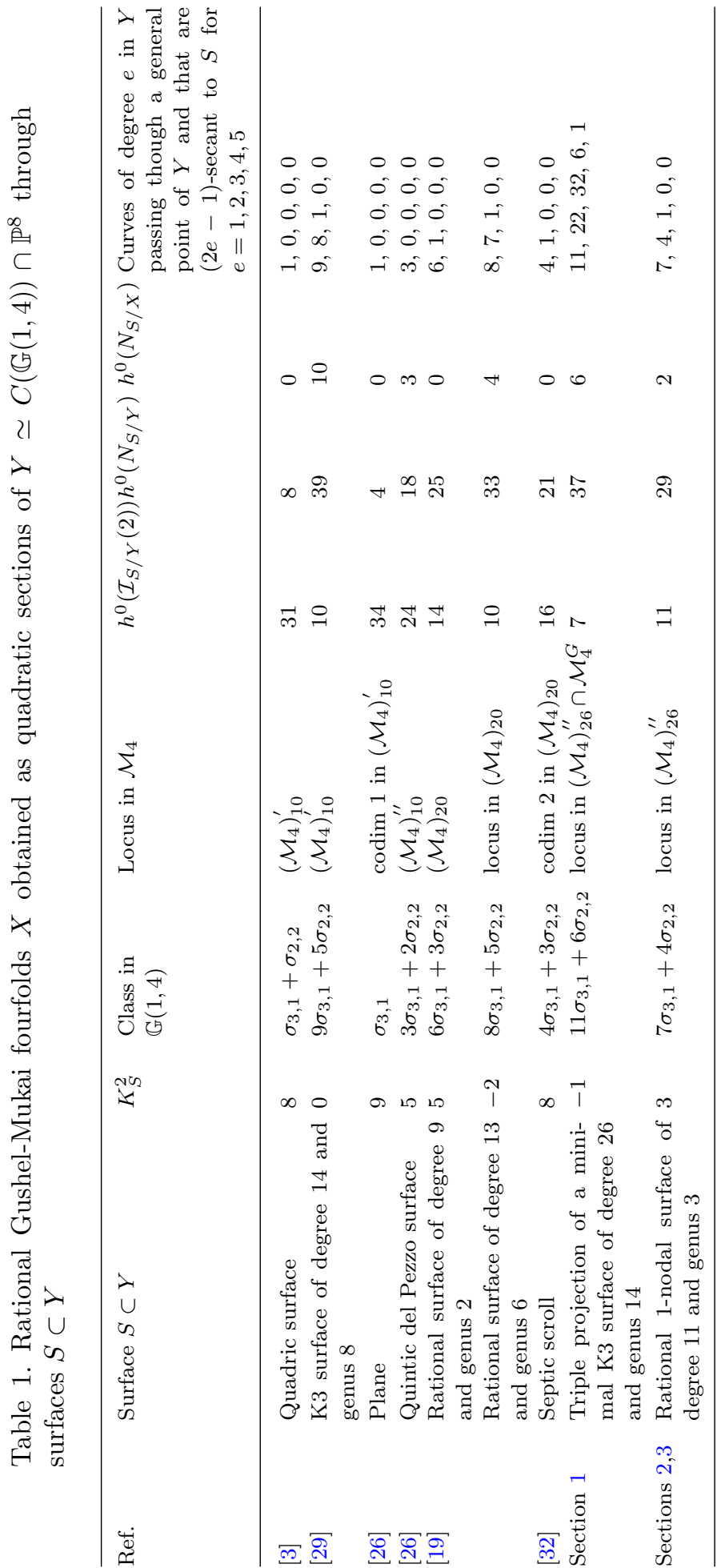




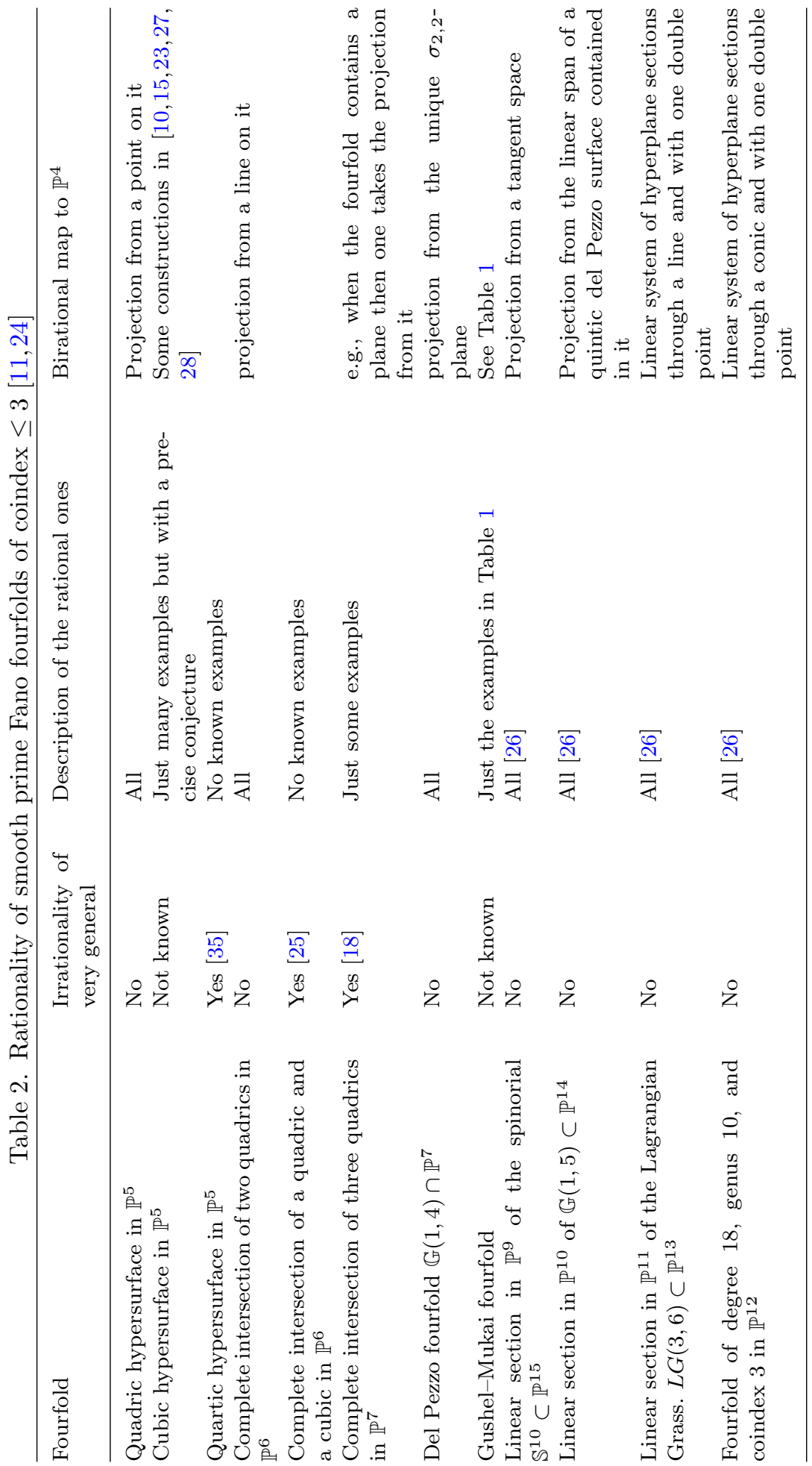


In Sect. 1.6, we describe an explicit birational map between $X$ and the cubic fourfold $C$.

\subsection{Rationality of Cubic Fourfolds in $\mathcal{C}_{26}$}

Here we recall some results from [27,28] (see also [29]) about the rationality of special cubic fourfolds of discriminant 26; see also [15-17] for general facts on cubic fourfolds.

Let $D \subset \mathbb{P}^{5}$ be a septimic surface with one node, which is the projection of a smooth del Pezzo surface of degree seven $D^{\prime} \subset \mathbb{P}^{7}$ from a general line intersecting the secant variety of $D^{\prime}$ at one point. A cubic fourfold $C \subset \mathbb{P}^{5}$ containing the surface $D$ has discriminant 26, and more precisely the locus $\mathcal{C}_{26}$ of cubic fourfolds of discriminant 26 can be described as the closure inside the moduli space $\mathcal{C}$ of cubic fourfolds of the locus of fourfolds containing such a surface.

The surface $D \subset \mathbb{P}^{5}$ admits a congruence of 5-secant conics: through a general point in $\mathbb{P}^{5}$ there passes a unique 5 -secant conic to $D$. Moreover, the linear system $\left|H^{0}\left(\mathcal{I}_{D, \mathbb{P} 5}^{2}(5)\right)\right|$ of hypersurfaces of degree 5 with points of multiplicity 2 along $D$ gives a dominant map

$$
\mathbb{P}^{5} \rightarrow Y_{0} \subset \mathbb{P}^{7}
$$

whose general fibers are the conic curves of the congruence, and where $Y_{0}$ is a smooth 4-dimensional linear section of $\mathbb{G}(1,4) \subset \mathbb{P}^{9}$. The restriction of the map (1.1) to a general cubic fourfold $C$ through $D$ induces a birational map $C \rightarrow Y_{0}$, whose inverse is defined by the linear system $\left|H^{0}\left(\mathcal{I}_{S_{0}, Y_{0}}^{2}(5)\right)\right|$ of hypersurfaces in $Y_{0}$ of degree 5 having points of multiplicity 2 along an irreducible surface $S_{0} \subset Y_{0} \subset \mathbb{P}^{7}$ of degree 17 and sectional genus 11 cut out by the 5 quadrics defining $Y_{0}$ and 13 cubics.

It turns out that $S_{0}$ is the projection of a surface $S \subset \mathbb{P}^{8}$ from a special point $p$ on the secant variety of $S$, where $S$ is a smooth surface of degree 17 , sectional genus 11 , cut out by 12 quadrics, and isomorphic to a triple projection of a minimal K3 surface of degree 26 in $\mathbb{P}^{14}$. The equations of $S$ can be determined from those of $S_{0}$ using the package IntegralClosure [8]; see also the function associatedK3surface from the package SpecialFanoFourfolds [34], which does most of this automatically.

\subsection{Gushel Fourfolds in $\left(\mathcal{M}_{4}\right)_{26}^{\prime \prime}$ : Construction of a Triple $S \subset X \subset Y$}

Continuing from the previous subsection, let $\nu_{p}: \mathbb{P}^{8} \rightarrow-\mathbb{P}^{7}$ denote the projection from the point $p$ such that $\nu_{p}(S)=S_{0}$, and let $Y=\overline{\nu_{p}^{-1}\left(Y_{0}\right)}$ be the cone over $Y_{0}$ of vertex $p$. Let $X \subset Y$ be a general quadratic section of $Y$ containing $S$. Then $X$ is a smooth Gushel fourfold which belongs to $\left(\mathcal{M}_{4}\right)_{26}^{\prime \prime}$.

Indeed, the surface $S_{0} \subset Y_{0} \subset \mathbb{G}(1,4)$, which is equal to the image of $S$ via the Gushel map of $X$, has class $11 \sigma_{3,1}+6 \sigma_{2,2}$ in the Chow ring of $\mathbb{G}(1,4)$, as one can verify by simple calculations with Schubert cycles. Then, from $(0.1)$ and $(0.2)$ it follows that $(S)_{X}^{2}=37$ and $[X] \in\left(\mathcal{M}_{4}\right)_{26}^{\prime \prime}$, since one has $\operatorname{deg}(S)=17, g(S)=11, \chi\left(\mathcal{O}_{S}\right)=2$, and $K_{S}^{2}=-1$. This calculation can be performed automatically using the functions discriminant and describe from the package SpecialFanoFourfolds. 


\subsection{Count of Parameters from the Triple $S \subset X \subset Y$}

Let $N_{S / X}$ and $N_{S / Y}$ denote, respectively, the normal bundle of the surface $S$ in $X$, and of $S$ in $Y$. A Macaulay2 calculation tells us that $h^{1}\left(N_{S / Y}\right)=0$, $h^{0}\left(N_{S / Y}\right)=37$, and $h^{0}\left(N_{S / X}\right)=6$. It follows that there exists a unique irreducible component $\mathcal{S}$ of the Hilbert scheme of $Y$ which contains $[S]$, and $\mathcal{S}$ is smooth at $[S]$ of dimension 37. Since we have $h^{0}\left(\mathcal{I}_{S, Y}(2)\right)=7$ (and this value is minimal on $\mathcal{S}$ ), we deduce by the same semicontinuity argument explained in [32, Subsection 1.5] that inside the 39-dimensional projective space $\mathbb{P}\left(H^{0}\left(\mathcal{O}_{Y}(2)\right)\right)$ of quadratic sections of $Y$ the family of fourfolds containing a surface in $\mathcal{S}$ has codimension at most $39-(37+(7-1)-6)=2$. This calculation can be performed automatically using the function parameterCount from the package SpecialFanoFourfolds.

\subsection{Congruence of 9-Secant Quintic Curves to $S \subset Y$}

We claim that the surface $S$ admits inside $Y$ a congruence of 9 -secant rational normal quintic curves: through a general point of $Y$ there passes a unique rational normal quintic curve which is 9 -secant to $S$ and contained in $Y$.

This can be verified by considering the rational map $\phi: Y \rightarrow \rightarrow \mathbb{P}^{6}$ defined by the linear system $\left|H^{0}\left(\mathcal{I}_{S, Y}(2)\right)\right|$ of quadratic sections through $S$, which is birational onto a non-normal sextic hypersurface $Z \subset \mathbb{P}^{6}$. If $p \in Y$ is a general point, then through the point $\phi(p) \in Z$ there pass 72 lines that are contained in $Z$. These 72 lines come from $(2 e-1)$-secant curves to $S$ of degree $e \geq 1$ which pass through $p$ and are contained in $Y$. Denoting by $n_{e}$ the number of such degree-e curves, we have $n_{1}=11, n_{2}=22, n_{3}=32, n_{4}=6, n_{5}=1$, and $n_{e}=0$ for $e \geq 6$. This calculation can be performed automatically using the function detectCongruence from the package SpecialFanoFourfolds.

Alternately, using tools from the package Cremona, one verifies that the linear system $\left|H^{0}\left(\mathcal{I}_{S, Y}^{5}(9)\right)\right|$ of hypersurfaces in $Y$ of degree 9 with points of multiplicity 5 along $S$ gives a dominant rational map

$$
Y \rightarrow X^{\prime} \subset \mathbb{P}^{8}
$$

whose general fibers are rational normal quintic curves, the curves of the congruence to $S$. The image $X^{\prime}$ is a Gushel fourfold, a smooth quadratic section of a cone $Y^{\prime} \subset \mathbb{P}^{8}$ over a smooth 4-dimensional linear section of $\mathbb{G}(1,4)$.

The restriction of the map (1.2) to $X$ induces a birational map

$$
Y \supset X \stackrel{\sim}{\sim} \rightarrow X^{\prime} \subset Y^{\prime}
$$

whose inverse is of the same type, i.e, it is the restriction to $X^{\prime}$ of the rational map $Y^{\prime} \rightarrow X$ defined by the linear system of hypersurfaces in $Y^{\prime}$ of degree 9 with points of multiplicity 5 along a smooth triple projection $S^{\prime} \subset Y^{\prime}$ of a minimal K3 surface of degree 26. In particular, the Gushel fourfold $X^{\prime}$ also belongs to $\left(\mathcal{M}_{4}\right)_{26}^{\prime \prime}$. 


\subsection{Rationality of $X$ from the Rationality of a Particular Rational Singular Fourfold $\hat{X}$ with $S \subset \hat{X} \subset Y$}

Let $\hat{X} \subset Y$ be a general quadratic section of $Y$ containing the surface $S$ and the vertex $p$ of $Y$. Then $\hat{X}$ has $p$ as the only singularity and the restriction of the projection from $p$ induces a birational map $\hat{X}-\rightarrow Y_{0}$, whose inverse is defined by the quadrics through a minimal K3 surface $F$ of degree 10. Since $Y_{0}=\mathbb{G}(1,4) \cap \mathbb{P}^{7}$ is rational (indeed, the projection from the unique $\sigma_{2,2}$-plane contained in it gives a birational map onto $\left.\mathbb{P}^{4}\right)$, we have that $\hat{X}$ is rational. On the other hand, the restriction of the map (1.2) induces a birational map

$$
Y \supset \hat{X} \stackrel{\sim}{\sim} \rightarrow X^{\prime} \subset Y^{\prime}
$$

due to the fact that $\hat{X}$ is transversal to the congruence to $S$ : the quintic curve of the congruence passing through a general point of $\hat{X}$ is not contained in $\hat{X}$. Therefore we deduce that also $X^{\prime}$ and hence $X$ are rational.

\subsection{Summary Construction}

Summing up we have the following diagram of birational maps, connecting explicitly general cubic fourfolds in $\mathcal{C}_{26}$ to Gushel fourfolds in $\left(\mathcal{M}_{4}\right)_{26}^{\prime \prime}$ :

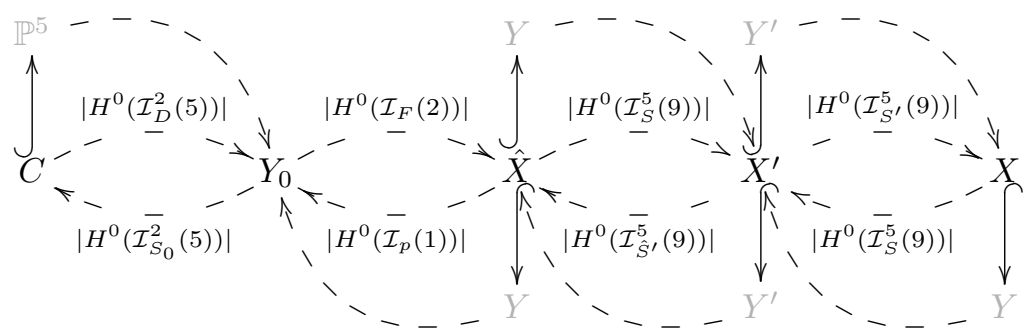

where $[C] \in \mathcal{C}_{26}$ is a general cubic fourfold of discriminant 26 containing a septimic one-nodal del Pezzo surface $D ;[X],\left[X^{\prime}\right] \in\left(\mathcal{M}_{4}\right)_{26}^{\prime \prime} \cap \mathcal{M}_{4}^{G}$ are (smooth) Gushel fourfolds of discriminant 26 contained, respectively, in cones $Y$ and $Y^{\prime}$ over $Y_{0}=\mathbb{G}(1,4) \cap \mathbb{P}^{7} ; \hat{X} \subset Y$ is a Gushel fourfold singular at $p$, where $p$ is the vertex of $Y ; S \subset X \cap \hat{X}$ and $S^{\prime}, \hat{S}^{\prime} \subset X^{\prime}$ are smooth surfaces, isomorphic to triple projections of minimal K3 surfaces of degree 26 (the intersection $S^{\prime} \cap \hat{S}^{\prime}$ consists of a twisted cubic curve and 31 points); $S_{0} \subset Y_{0}$ is the projection of $S$ from $p$; and $F \subset Y_{0}$ is a minimal K3 surface of degree 10 .

\subsection{Ancillary Files}

We provide an ancillary file, named gushel26. $2,{ }^{1}$ containing explicit equations for an example of map as (1.3) over the finite field $\mathbb{F}_{10000019}$ (this is only necessary to reduce the size of the file). After loading that file in Macaulay2, some variables will be defined as follows:

$X, X^{\prime}$ two instances of the type SpecialGushelMukaiFourfold, respectively, the source and target of the map (1.3);

\footnotetext{
${ }^{1}$ It is available at https://github.com/giovannistagliano/RationalGushelFourfolds.
} 
psi, psi' two instances of the type RationalMap, respectively, the birational map (1.3) and its inverse (psi' is the same that inverse psi);

Psi, Psi' two instance of the type RationalMap, respectively, the dominant rational map (1.2) from $Y$ to $X^{\prime}$ that extends psi, and the analogous map from $Y^{\prime}$ to $X$ that extends psi'.

For technical details about these types of data, we refer to the documentation of the packages Cremona [31] and SpecialFanoFourfolds [34]. We now show how to load the file and extract some basic information from it (some output lines are omitted for brevity).

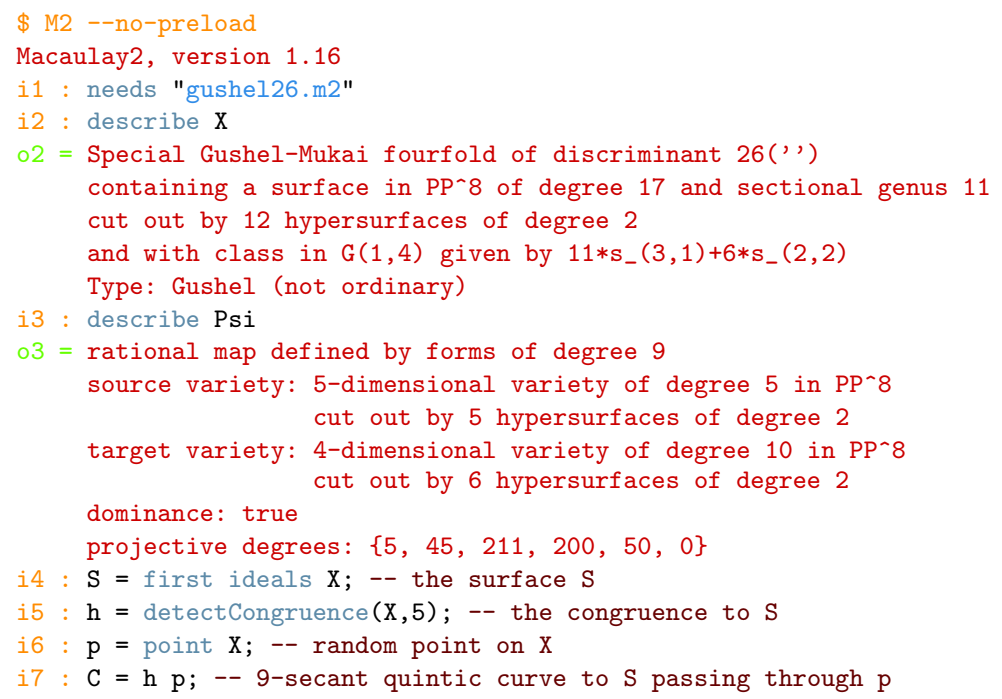

\section{Rationality Without Passing Through Singular Fourfolds}

A count of parameters shows that a general triple projection of a general minimal K3 surface of degree 26 is contained in a one-dimensional family of 5 -dimensional linear sections of cones $C(\mathbb{G}(1,4)) \subset \mathbb{P}^{10}$. So one expects that the Gushel fourfolds constructed in Sect. 1 can be deformed to ordinary fourfolds. Anyway, the simple argument given in Sect. 1.5 to deduce the rationality of the fourfold $X$ is not available in the ordinary case. In this section, we remedy this with a construction that does not involve singular Gushel fourfolds.

Indeed, keeping the notation as in Sect. 1, here we construct a special Gushel fourfold $\tilde{X}$ with $S \subset \tilde{X} \subset Y$, uniquely determined by the embedding $S \subset Y$, which turns out to be smooth and transversal to the congruence of 9secant quintic curves to $S$. We then show that $\tilde{X}$ contains another "simpler" surface $T$ which admits inside $Y$ a congruence of 5 -secant cubic curves. So we deduce that $\tilde{X}$ is rational, and hence that $X$ is rational. 


\subsection{Construction of $\tilde{X}$ and of a Dominant Map $\tilde{X} \rightarrow \mathbb{P}^{2}$}

Consider again the birational map introduced in Sect. 1.4,

$$
\phi: Y \rightarrow Z \subset \mathbb{P}^{6},
$$

defined by the linear system $\left|H^{0}\left(\mathcal{I}_{S, Y}(2)\right)\right|$, where $Z=\overline{\phi(Y)} \subset \mathbb{P}^{6}$ is a sextic hypersurface. We take $\tilde{X}$ to be the top dimensional component of the (closure of the) exceptional locus of $\phi$, and the map $\tilde{X} \rightarrow \mathbb{P}^{2}$ to be defined by the quadrics through the (closure of the) union of all 4 -secant conics to $S$ contained in $\tilde{X}$. Let us provide some more detail.

2.1.1. The Fourfold $\tilde{\boldsymbol{X}}$. Using tools from the package Cremona, one verifies that the base locus scheme of the inverse map $\phi^{-1}: Z \rightarrow Y$ is the union of the following components:

- a smooth cubic fourfold $\tilde{C} \subset \mathbb{P}^{5} \subset \mathbb{P}^{6}$ (with $[\tilde{C}] \in \mathcal{C}_{26}$, see Remark 2.2); the fiber of $\phi$ at a general point of $\tilde{C}$ consists of two points;

- a smooth cubic scroll surface $\Sigma_{3} \subset \mathbb{P}^{4} \subset \mathbb{P}^{6}$, which is double; the fiber of $\phi$ at a general point of $\Sigma_{3}$ is an irreducible conic curve which is 4 -secant to $S$;

- a surface of degree 46 cut out in $\mathbb{P}^{6}$ by 81 quintic hypersurfaces; the fiber of $\phi$ at a general point of this surface is a 2-secant line to $S$.

Then, the fourfold $\tilde{X}$ is taken to be $\overline{\phi^{-1}(\tilde{C})}$, so that the restriction of $\phi$ induces a generically finite map of degree 2 from $\tilde{X}$ to $\tilde{C}$. One sees that $\tilde{X}$ is a smooth Gushel fourfold which is transversal to the congruence to $S$ and in particular it is birational to $X^{\prime}$ via the restriction of the map (1.2). Moreover, the inverse map $X^{\prime} \rightarrow \tilde{X}$ is defined once again by the linear system $\left|H^{0}\left(\mathcal{I}_{\tilde{S}^{\prime}}^{5}(9)\right)\right|$, where $\tilde{S}^{\prime} \subset X^{\prime} \subset Y^{\prime}$ is a smooth triple projection of a minimal K3 surface of degree 26. We stress that this surface $\tilde{S}^{\prime} \subset Y^{\prime}$ and the fourfold $\tilde{X}$ are uniquely determined by the embedding $S \subset Y$.

2.1.2. The Map $\tilde{\boldsymbol{X}} \rightarrow \mathbb{P}^{\mathbf{2}}$. The intersection $\tilde{C} \cap \Sigma_{3}$ is a twisted cubic curve, and the fiber of $\phi$ at a general point of this curve is a 4-secant conic curve to $S$ contained in $\tilde{X}$. The inverse image $R=\overline{\phi^{-1}\left(\tilde{C} \cap \Sigma_{3}\right)}$ is an irreducible surface contained in $\tilde{X}$ of degree 17 and sectional genus 6 cut out in $\mathbb{P}^{8}$ by 9 quadrics and 7 cubics. Let

$$
\eta: Y \rightarrow \mathbb{P}^{3}
$$

be the rational map defined by the linear system $\left|H^{0}\left(\mathcal{I}_{R, Y}(2)\right)\right|$ of quadratic sections of $Y$ through $R$. Since $R \subset \tilde{X}$, the restriction of $\eta$ induces another map

$$
\left.\eta\right|_{\tilde{X}}: \tilde{X} \rightarrow \mathbb{P}^{2} \subset \mathbb{P}^{3} .
$$

One sees that $\eta$ (resp., $\left.\eta\right|_{\tilde{X}}$ ) is a dominant map, whose general fibers are surfaces like $S$, that is, smooth triple projections of minimal K3 surfaces of degree 26. Moreover the surface $S$ is recovered as a special fiber, and all these fibers share the same twisted cubic curve contained in $S$. 
Remark 2.1. The projection of the surface $S$ from the plane spanned by a general 4-secant conic to $S$ contained in $Y$ (computable as the fiber of $\phi$ at a general point on $\Sigma_{3}$ ) is a smooth surface in $\mathbb{P}^{5}$ of degree 13 and sectional genus 11 cut out by 6 cubics. This surface in $\mathbb{P}^{5}$ admits a congruence of 14 -secant rational normal quintic curves from which one can deduce the rationality for cubic fourfolds of discriminant 26. (See also the example in [28, Table 1, row 15] of a nodal surface in $\mathbb{P}^{5}$.)

\subsection{Special Fibers of the Map $\tilde{X} \rightarrow \mathbb{P}^{2}$ : Construction of a Surface $T \subset$ $\tilde{\boldsymbol{X}} \subset \boldsymbol{Y}$}

The 3-dimensional projective space, image of the map $\eta$, contains a special plane $\Pi$ which intersects $\overline{\eta(\tilde{X})} \simeq \mathbb{P}^{2}$ along a line $L$. The fiber of $\eta$ at a general point of $L$ (as well as of $\Pi$ ) is an irreducible rational surface $T \subset Y \subset \mathbb{P}^{8}$ of degree 11 , sectional genus 3 , cut out in $\mathbb{P}^{8}$ by 16 quadrics, having smooth normalization and a node as the only singularity. In the Chow ring of $\mathbb{G}(1,4)$ we have $\left[\gamma_{\tilde{X}}(T)\right]=7 \sigma_{3,1}+4 \sigma_{2,2}$, so from $(0.1)$ it follows that $(T)_{\tilde{X}}^{2}=19$ (this is also confirmed from the fact that two fibers of $\eta$ corresponding to two general points of $L$ intersects at 19 points), and (0.2) tells us that any smooth quadratic section of $Y$ containing $T$ is a Gushel fourfold of discriminant 26, hence corresponding to a point of $\left(\mathcal{M}_{4}\right)_{26}^{\prime \prime}$.

From another point of view, using the map $\eta$, we are able to obtain a degeneration of the surface $S$ as the union of the surface $T$ and a smooth surface $Q$ of degree 6 and sectional genus 2 with $\left[\gamma_{\tilde{X} *}(Q)\right]=2\left(2 \sigma_{3,1}+\sigma_{2,2}\right)=$ $2 \sigma_{2} \sigma_{1}^{2}$ and such that the intersection $T \cap Q$ is an irreducible curve of degree 7 with $p_{a}=2$ and $p_{g}=1$. This surface $Q$ is contained in the base locus of $\eta$. More precisely, the support of the base locus of $\eta$ is the union of $Q$ with the surface $R$.

Remark 2.2. The plane $\Pi$ can be calculated as the image via $\eta$ of the 3dimensional linear space $\overline{\nu_{p}^{-1}(P)}$, where $\nu_{p}: Y \rightarrow Y_{0}$ is the projection from the vertex of $Y$, and $P$ is the unique $\sigma_{2,2}$ plane contained in $Y_{0}$. Moreover, the intersection $\overline{\nu_{p}^{-1}(P)} \cap \tilde{X}$ is a quadric surface which is sent birationally by $\phi$ to a one-nodal septimic surface $\tilde{D} \subset \tilde{C}$ as the surface $D \subset C$ considered in Sect. 1.1. In particular, we also deduce that $\tilde{C}$ is a cubic fourfold of discriminant 26 .

\subsection{Count of Parameters from the Triple $T \subset \tilde{X} \subset Y$}

As in Sect. 1.3, we compute with Macaulay2 that $h^{1}\left(N_{T / Y}\right)=0, h^{0}\left(N_{T / Y}\right)=$ 29 , and $h^{0}\left(N_{T / \tilde{X}}\right)=2$. Assuming that the Hilbert scheme Hilb $Y$ of $Y$ is smooth at $[T]$ (which is reasonable, but not guaranteed since $T$ is not a local complete intersection), we have that $\mathrm{Hilb}_{Y}$ contains a unique irreducible component $\mathcal{T}$ which contains $[T]$, and the dimension of $\mathcal{T}$ is 29. Therefore, since we have $h^{0}\left(\mathcal{I}_{T, Y}(2)\right)=11$ and this value is minimal, we deduce that inside the projective space $\mathbb{P}\left(H^{0}\left(\mathcal{O}_{Y}(2)\right)\right)$ of quadratic sections of $Y$ the family of fourfolds containing a surface in $\mathcal{T}$ has codimension at most $39-(29+(11-1)-2)=2$. 


\subsection{Congruence of 5-Secant Cubic Curves to $T \subset Y$ and Rationality of $\tilde{X}$}

The surface $T \subset Y$ admits inside $Y$ a congruence of 5-secant twisted cubic curves, and $\tilde{X}$ is transversal to this congruence.

Indeed one verifies that the linear system $\left|H^{0}\left(\mathcal{I}_{T, Y}^{3}(5)\right)\right|$ of hypersurfaces in $Y$ of degree 5 with points of multiplicity 3 along $T$ gives a dominant rational map

$$
Y \rightarrow W \subset \mathbb{P}^{10}
$$

onto a smooth 4-dimensional linear section $W$ of $\mathbb{G}(1,5) \subset \mathbb{P}^{14}$, and whose general fibers are twisted cubic curves. The restriction of $(2.3)$ to $\tilde{X}$ induces a birational map

$$
Y \supset \tilde{X} \stackrel{\simeq}{\simeq} W \subset \mathbb{P}^{10}
$$

whose inverse is defined by the linear system of hypersurfaces of degree 5 with points of multiplicity 3 along a smooth surface $U \subset W$ of degree 21 and sectional genus 13, isomorphic to a double projection of a simple projection of a minimal K3 surface of degree 26.

We deduce the rationality of $\tilde{X}$ from that of $W$. Indeed, $W$ must contain a quintic del Pezzo surface, and it is classically known that the linear system of hyperplanes through this surface gives a birational map $W \rightarrow \mathbb{P}^{4}$.

The congruence to $T \subset Y$ can be also verified by considering the map $Y \rightarrow \mathbb{P}^{10}$, defined by the linear system $\left|H^{0}\left(\mathcal{I}_{T, Y}(2)\right)\right|$ of quadratic sections of $Y$ through $T$, which turns out to be birational onto a fivefold of degree 20 cut out by 7 quadrics. Through the general point of this fivefold there pass 12 lines, which come from seven 1 -secant lines to $T$, four 3 -secant conics to $T$, and one single 5 -secant twisted cubic to $T$.

There is a further way to find the congruence to $T \subset Y$. Indeed, one has that the reducible surface $T \cup Q$ considered in Sect. 2.2, which is a degeneration of the surface $S$, admits a congruence of 9 -secant quintic curves, exactly as $S$ does. In this degenerate case, the curves of the congruence split into 4-secant conics to $T \cup Q$ and 5 -secant twisted cubics to $T$.

\subsection{Summary Construction}

With the notation above introduced, we have the following diagram involving cubic fourfolds in $\mathcal{C}_{26}$ and Gushel fourfolds in $\left(\mathcal{M}_{4}\right)_{26}^{\prime \prime}$, and where all the fourfolds are smooth.

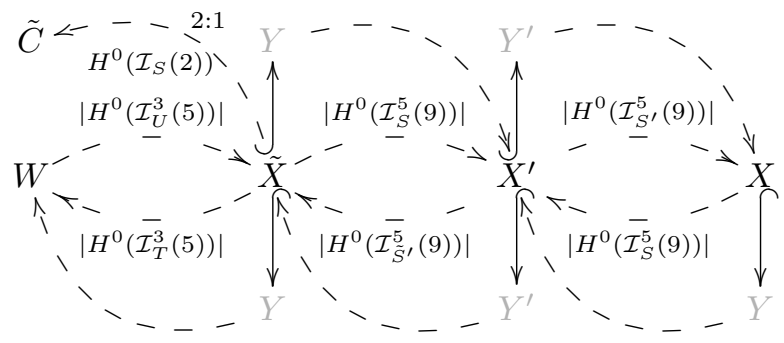




\subsection{Ancillary Files}

Continuing from Sect. 1.7, after the loading of the ancillary file in Macaulay2, some other variables are defined as following:

Xtilde an instance of the type SpecialGushelMukaiFourfold, the Gushel fourfold corresponding to the pair $(T, \tilde{X})$ constructed above;

Eta, eta two instances of the type RationalMap, respectively, the map $Y \rightarrow \mathbb{P}^{3}$ in $(2.1)$ and its restriction $\tilde{X} \rightarrow \mathbb{P}^{2} \subset \mathbb{P}^{3}$ in (2.2).

Psi2, psi2 two instances of the type RationalMap, respectively, the map $Y \rightarrow W$ in (2.3) and its restriction $\tilde{X} \rightarrow W$ in (2.4).

By way of example, we now compute a general and a special fiber of the map $(2.2)$.

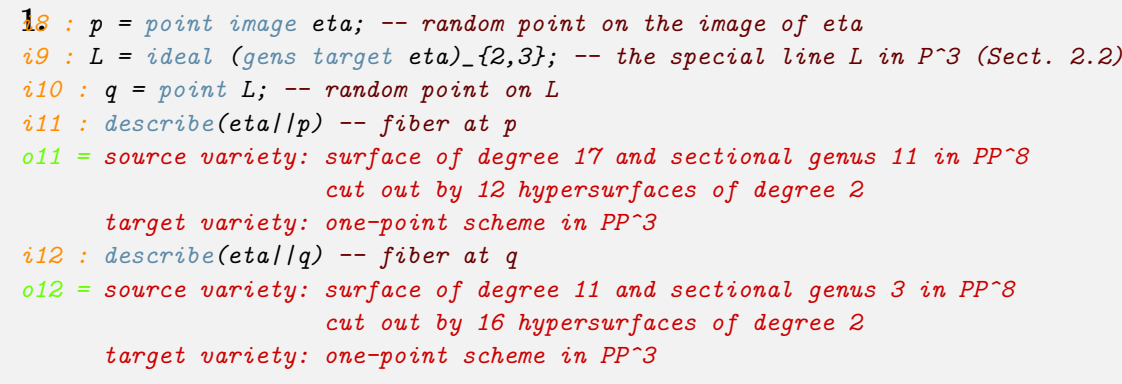

\section{Construction of New Rational Mukai Fourfolds}

In this section, we briefly explain how using the same method given in [32], one can directly construct a rational one-nodal surface $T \subset Y$ of degree 11 and sectional genus 3 as the one constructed in Sect. 2. Actually, we do better than this: We are able to construct a rational one-nodal surface $\breve{T}$ of degree 11 and sectional genus 3 inside a smooth hyperplane section $\breve{Y}$ of $\mathbb{G}(1,4)$. This leads us to find out rational ordinary fourfolds in $\left(\mathcal{M}_{4}\right)_{26}^{\prime \prime}$.

\subsection{Construction of $\breve{T} \subset \breve{Y}$}

Let $E^{\prime} \subset \mathbb{P}^{6}$ be the image of the plane via the linear system of quartic curves with one double point $q_{0}$ and 5 simple base points $q_{1}, \ldots, q_{5}$. Then $E^{\prime}$ is a smooth surface of degree 7 and sectional genus 2 cut out by 8 quadrics. ${ }^{2}$ Let $E \subset \mathbb{P}^{5}$ be the projection of $E^{\prime}$ from a general point on the secant variety of $E^{\prime}$. Then $E$ is an irreducible surface of degree 7 , sectional genus 2 , cut out by 2 quadrics and 5 cubics, with a single node as the only singularity, and having normalization isomorphic to $E^{\prime}$. Take $B \subset \mathbb{P}^{5}$ to be a general smooth cubic scroll surface which cuts $E$ along a quintic elliptic curve (such a curve

\footnotetext{
${ }^{2}$ The surface $E^{\prime} \subset \mathbb{P}^{6}$ is a hyperplane section of a so-called Edge variety [7]. This surface also occurs in the classification of special birational transformations, see [33, Table 4, Case VI].
} 
is obtained as the image on $E^{\prime}$ and hence on $E$ of a general plane cubic curve passing through the six base points $q_{0}, \ldots, q_{5}$ ).

The linear system of quadrics through $B$ defines a birational map

$$
\mathbb{P}^{5}-\rightarrow \breve{Y} \simeq \mathbb{G}(1,4) \cap \mathbb{P}^{8} \subset \mathbb{P}^{8}
$$

onto a smooth hyperplane section $\breve{Y}$ of $\mathbb{G}(1,4) \subset \mathbb{P}^{9}$. The restriction of this map to $E$ induces an isomorphism between $E$ and a surface $\breve{T} \subset \breve{Y}$.

This surface $\breve{T} \subset \breve{Y}$ shares all the properties of the surface $T \subset Y$ constructed in Sect. 2. In particular, $\breve{T} \subset \breve{Y}$ is a one-nodal irreducible surface of degree 11 , sectional genus 3 , cut out in $\mathbb{P}^{8}$ by 16 quadrics, and having class $7 \sigma_{3,1}+4 \sigma_{2,2}$ in the Chow ring of $\mathbb{G}(1,4)$. A general quadratic section of $\breve{Y}$ through $\breve{T}$ gives an ordinary Gushel-Mukai fourfold in $\left(\mathcal{M}_{4}\right)_{26}^{\prime \prime}$, and such a fourfold is birational to a smooth 4-dimensional linear section of $\mathbb{G}(1,5) \subset \mathbb{P}^{14}$ via the linear system of quintic hypersurfaces with triple points along $\breve{T}$.

Remark 3.1. By counting parameters, one sees that the family of the reducible surfaces $B \cup E \subset \mathbb{P}^{5}$ has dimension 48 , and hence the family of the surfaces $\breve{T} \subset \breve{Y}$ obtained by this construction has dimension $48-35+15=$ $28<29=h^{0}\left(N_{\breve{T} / \breve{Y}}\right) ;$ see also [32, Remark 2.4].

Remark 3.2. Our ancillary file (see Sects. 1.7 and 2.6) also provides an explicit example of ordinary Gushel-Mukai fourfold corresponding to the pair $(\breve{T}, \breve{X})$, where $\breve{X}$ is a general quadratic section of $\breve{Y}$ through $\breve{T}$. The file includes the parameterization of $E^{\prime}$, the nodal projection of $E^{\prime}$ onto $E$, the map (3.1), the dominant map $\breve{Y} \rightarrow \mathbb{G}(1,5) \cap \mathbb{P}^{10}$ whose general fibers are 5-secant twisted cubic curves to $\breve{T}$, and the restriction of this map to $\breve{X}$ together with its inverse.

\section{Summary Table of Examples}

Table 1 summarizes some information about the Gushel-Mukai fourfolds known to be rational. It includes all the examples we found in the literature, the example constructed in the present paper, and some few others of which we omit the details. In all cases, except the 4 th, the rationality of the fourfold follows from a congruence of $(2 e-1)$-secant curves of degree $e \geq 1$.

\section{Acknowledgements}

The author has benefited from discussions with Michele Bolognesi, Olivier Debarre, and Francesco Russo.

Funding Open access funding provided by Università degli Studi di Catania within the CRUI-CARE Agreement.

Open Access. This article is licensed under a Creative Commons Attribution 4.0 International License, which permits use, sharing, adaptation, distribution and reproduction in any medium or format, as long as you give appropriate credit to the original author(s) and the source, provide a link to the Creative Commons 
licence, and indicate if changes were made. The images or other third party material in this article are included in the article's Creative Commons licence, unless indicated otherwise in a credit line to the material. If material is not included in the article's Creative Commons licence and your intended use is not permitted by statutory regulation or exceeds the permitted use, you will need to obtain permission directly from the copyright holder. To view a copy of this licence, visit http:// creativecommons.org/licenses/by/4.0/.

Publisher's Note Springer Nature remains neutral with regard to jurisdictional claims in published maps and institutional affiliations.

\section{References}

[1] Addington, N., Thomas, R.: Hodge theory and derived categories of cubic fourfolds. Duke Math. J. 163(10), 1886-1927 (2014)

[2] Bolognesi, M., Russo, F., Staglianò, G.: Some loci of rational cubic fourfolds. Math. Ann. 373(1), 165-190 (2019)

[3] Debarre, O., Iliev, A., Manivel, L. Special prime Fano fourfolds of degree 10 and index 2. In: Hacon, C., Mustaţă, M., Popa, M. (eds.) Recent Advances in Algebraic Geometry: A Volume in Honor of Rob Lazarsfeld's 60th Birthday. London Mathematics Society. Lecture Note Series, pp. 123-155. Cambridge University Press (2015)

[4] Debarre, O., Kuznetsov, A.: Gushel-Mukai varieties: classification and birationalities. Algebr. Geom. 5, 15-76 (2018)

[5] Debarre, O., Kuznetsov, A.: Gushel-Mukai varieties: linear spaces and periods. Kyoto J. Math. 59(4), 897-953 (2019)

[6] Debarre, O., Kuznetsov, A.: Gushel-Mukai varieties: moduli. Int. J. Math. 31(2) (2020)

[7] Edge, W.L.: The number of apparent double points of certain loci. Math. Proc. Camb. Philos. Soc. 28(3), 285-299 (1932)

[8] Eisenbud, D., Stillman, M., Taylor, A.: IntegralClosure: a MACAUlay2 package for computing integral closures of affine domains and ideals, version 1.09, source code. https://github.com/Macaulay2/M2/blob/development/M2/Macaulay2/ packages/IntegralClosure.m2 (2020)

[9] Enriques, F.: Sulla irrazionalità da cui può farsi dipendere la resoluzione di un'equazione algebrica $f(x, y, z)=0$ con funzioni razionali di due parametri. Math. Ann. 49, 1-23 (1897)

[10] Fano, G.: Sulle forme cubiche dello spazio a cinque dimensioni contenenti rigate razionali del $4^{\circ}$ ordine. Comment. Math. Helv. 15(1), 71-80 (1943)

[11] Fujita, T.: Classification theories of polarized varieties. In: London Mathematics Society Lecture Note Series, vol. 155. Cambridge University Press, Cambridge (1990)

[12] Fulton, W.: Intersection theory. In: Ergebnisse der Mathematik und ihrer Grenzgebiete(3), no. 2. Springer (1984)

[13] Grayson, D.R., Stillman, M.E.: Macaulay2 - a software system for research in algebraic geometry (version 1.16). http://www.math.uiuc.edu/Macaulay2/ (2020) 
[14] Gushel, N.P.: Fano varieties of genus 6 (in Russian). Izv. Akad. Nauk USSR Ser. Mat. 46(6), 1159-1174 (1982) (English transl.: Math. USSR-Izv. 21(3), 445-459 (1983))

[15] Hassett, B.: Some rational cubic fourfolds. J. Algebraic Geom. 8(1), 103-114 (1999)

[16] Hassett, B.: Special cubic fourfolds. Comp. Math. 120(1), 1-23 (2000)

[17] Hassett, B.: Cubic fourfolds, K3 surfaces, and rationality questions. In: Pardini, R., Pirola, G. P. (eds.) Rationality Problems in Algebraic Geometry: Levico Terme, Italy 2015, pp. 29-66. Springer, Cham (2016)

[18] Hassett, B., Pirutka, A., Tschinkel, Y.: Intersections of three quadrics in $\mathbb{P}^{7}$. Surv. Differ. Geom. 22, 259-274 (2017)

[19] Hoff, M., Staglianò, G.: New examples of rational Gushel-Mukai fourfolds. Math. Z. 296, 1585-1591 (2020)

[20] Kontsevich, M., Tschinkel, Y.: Specialization of birational types. Invent. Math. 217(2), 415-432 (2019)

[21] Kuznetsov, A.: Derived categories of cubic fourfolds. In: Cohomological and Geometric Approaches to Rationality Problems. Progress in Mathematics, vol. 282. Birkhäuser Boston, pp. 219-243 (2010)

[22] Kuznetsov, A.: Derived categories view on rationality problems. In: Pardini, R., Pirola, G. P. (eds.)Rationality Problems in Algebraic Geometry: Levico Terme, Italy 2015, pp. 67-104. Springer, Cham (2016)

[23] Morin, U.: Sulla razionalità dell'ipersuperficie cubica dello spazio lineare $S_{5}$. Rend. Semin. Mat. Univ. Padova 11, 108-112 (1940)

[24] Mukai, S.: Biregular classification of Fano 3-folds and Fano manifolds of coindex 3. Proc. Natl. Acad. Sci. USA 86(9), 3000-3002 (1989)

[25] Nicaise, J., Ottem, J.C.: Tropical degenerations and stable rationality. Preprint: arxiv:1911.06138 (2019)

[26] Roth, L.: Algebraic varieties with canonical curve sections. Ann. Mat. Pura Appl. 29(1), 91-97 (1949)

[27] Russo, F., Staglianò, G.: Congruences of 5-secant conics and the rationality of some admissible cubic fourfolds. Duke Math. J. 168(5), 849-865 (2019)

[28] Russo, F., Staglianò, G.: Trisecant flops, their associated K3 surfaces and the rationality of some Fano fourfolds. Preprint: https://arxiv.org/abs/1909.01263 (2019)

[29] Russo, F., Staglianò, G.: Explicit rationality of some cubic fourfolds. In: The Proceeding of the Schiermonnikoog Conference Rationality of Algebraic Varieties (2020) (to appear)

[30] Shepherd-Barron, N.I.: The rationality of quintic del Pezzo surfaces - a short proof. Bull. Lond. Math. Soc. 24(3), 249-250 (1992)

[31] Staglianò, G.: A Macaulay2 package for computations with rational maps. J. Softw. Alg. Geom. 8(1), 61-70 (2018)

[32] Staglianò, G.: On some families of Gushel-Mukai fourfolds. Preprint: https:// arxiv.org/abs/2002.07026 (2020)

[33] Staglianò, G.: Special cubic birational transformations of projective spaces. Collect. Math. 71, 123-150 (2020)

[34] Staglianò, G.: SpecialFanoFourfolds: a macaulay2 package for working with special cubic fourfolds and special Gushel-Mukai fourfolds, version 0.9.5, source 
code. https://github.com/Macaulay2/M2/tree/development/M2/Macaulay2/ packages/SpecialFanoFourfolds.m2 (2020)

[35] Totaro, B.: Hypersurfaces that are not stably rational. J. Am. Math. Soc. 29(3), 883-891 (2015)

Giovanni Staglianò

Dipartimento di Matematica e Informatica

Università degli Studi di Catania

Catania

Italy

e-mail: giovannistagliano@gmail.com

Received: April 3, 2020.

Revised: November 3, 2020.

Accepted: May 28, 2021. 